\title{
PREDOMINANCIA ECOLÓGICA Y TAXONÓMICA DE NOMBRES DE AVES ENTRE ESTUDIANTES UNIVERSITARIOS PERUANOS
}

\author{
Taxonomic and ecological salience of bird folk names among Peruvian undergraduated \\ students
}

José PIZARRO NEYRA ${ }^{1} *$

${ }^{1}$ Asociación para el Desarrollo de las Ciencias Biológicas en el Perú; Calle Arica 176, Tacna, Perú. *josepizarroneyra@gmail.com

Submitted: 24/09/2020; Accepted: 08/12/2020; Published: 10/01/2021

\section{RESUMEN}

Durante octubre y noviembre de 2019, 340 estudiantes de la Universidad Nacional Jorge Basadre de Tacna, Perú, participaron en la colecta de nombres populares de aves mediante la técnica de "freelisting". La información sirvió para ordenar los nombres populares de las aves mediante la estimación del índice de importancia relativa (IR) de cada nombre escrito en las listas. Luego, se construyeron dos índices con el IR: el índice de Predominancia Ecológica (ES) y el índice de Predominancia Taxonómica a nivel de orden (TS). Los resultados mostraron que los nombres con valores más altos de IR son aves domésticas, de valor de uso directo como alimento o mascotas, además de aves emblemáticas o fenotípicamente sobresalientes como el Cóndor andino, el Águila, el Gallito de las rocas, el Picaflor, el Avestruz y el Pelícano. Los valores del índice ES (ES=0.05) indican una pequeña predominancia de nombres de aves distribuidas localmente en las listas, probablemente por una escasa experiencia directa con aves por parte de los participantes. Los órdenes con valores del índice TS $>0.4$ corresponden a: Galliformes, Anseriformes, Cathartiformes, Psittaciformes, y Rheiformes. La mayor parte de ellas contienen espécies exóticas pero también aves que son comunes en zonas urbanas, como tres especies de loros, el gallinazo cabeza roja y los gorriones. Estos resultados confirman que entre los estudiantes universitarios peruanos son muy populares las aves exóticas así como aquellas aves que habitan el Perú pero que no se distribuyen localmente.

PALABRAS CLAVE: Predominancia ecológica, Predominancia taxonómica, Aves, Estudiantes universitarios, Perú.

\begin{abstract}
Using the free listing technique, bird folk names were gathered from 340 undergraduate students at the Jorge Basadre National University in Tacna, Peru during October-November 2019. The data collected were used to estimate the relative importance index (IR) of each bird name. Then, the IR index was used to calculate the indices of taxonomic salience (TS) and ecological salience (ES). The results reveals that the folk names of birds with high IR index scores corresponding to ones used as food or as pets, in addition to representing birds with phenotypic prominence such as Andean Cock on the rock, Andean Condor, Eagle, Hummingbird, Ostrich and Pelican. The ES index reaches a value of 0.06 and indicates a slight salience of local bird names, perhaps because the students have no direct experience with wild birds. Galliformes, Passeriformes, Anseriformes, Psittaciformes and, Rheiformes are the orders with a TS index $>0.4$, which contain mainly exotic species but too some common species in the city such as three species of parakeets, the turkey vulture or sparrows. The results confirm the popularity of exotic and non-locally distributed birds among Peruvian undergraduated students.
\end{abstract}

KEYWORDS: Ecological Salience, Taxonomic Salience, Undergraduated students, Bird folk names, Peru. 
Pizarro NEYRA, J. Predominancia ecológica y taxonómica de nombres de aves entre estudiantes universitarios peruanos. Ethnoscientia v. 6, n. 1, 2021. DOI: 10.22276/ethnoscientia.v6i1.353

\section{INTRODUCCIÓN}

La predominancia ecológica ha sido definida por HUNN (1999) como un índice de probabilidad de encuentros entre miembros de una comunidad humana y un grupo de organismos como función de la distribución espacio-temporal de las poblaciones de estos organismos. En nuestro caso se entiende que la distribución geográfica de las aves se presenta en el ambiente urbano, así como que el componente cultural proviene de la importancia relativa que las aves presentan para el habitante de la ciudad, lo que permite determinar su predominancia ecológica. La predominancia taxonómica en cambio es más difícil de definir en términos etnobiológicos, pero podría definirse en términos de la importancia relativa que alcanzan taxones diferentes en el vocabulario popular de un grupo humano. En nuestro caso hemos usado la suposición que hace DOUGERTHY (1978) acerca de los sistemas de clasificación etnobiológica, cuando disminuye la importancia de los animales para los grupos humanos urbanos, lo que hace crecer a las categorías de clasificación resultantes para incluir a cada vez más organismos. Este es el caso de los órdenes en la clasificación zoológica, que concentra todas las categorías infra-ordinales del taxón Neornithes. Es así que se determinan los órdenes más sobresalientes taxonómicamente, calculando la importancia relativa de las aves pertenecientes a este taxón.

Las aves son un grupo taxonómico de importancia en el Perú debido a que en el país existe una alta diversidad de ellas, contando con varias especies endémicas (PERÚ, 2018; BIRDLIFE INTERNATIONAL, 2020; SALINAS; ABARCA; WUST, 2018). Los estudios de los nombres locales de las aves pueden ayudar en el descubrimiento de nuevas especies de aves, en la descripción de comportamientos desconocidos para la ciencia formal, en el registro de nuevas ocurrencias, preparación de inventarios más eficientes y precisos, localización de especies amenazadas, y la corrección de posibles errores (FARIAS; ALVES, 2007).

\section{MATERIALES Y MÉTODOS}

\subsection{Sitio de estudio}

El estudio se realizó en la ciudad de Tacna (1800’00” LS 70¹5'13" LO) con 286.240 habitantes y localizada a 560 metros sobre el nivel del mar, cerca de la frontera entre Perú y Chile. Las principales actividades económicas del lugar son el comercio, el turismo y otros servicios conexos. Desde el punto de vista biogeográfico, la ciudad se encuentra en la provincia del Desierto del Pacífico y en una zona con especies endémicas de aves como 
Pizarro NEYRA, J. Predominancia ecológica y taxonómica de nombres de aves entre estudiantes universitarios peruanos. Ethnoscientia v. 6, n. 1, 2021. DOI: 10.22276/ethnoscientia.v6i1.353

Eulidia yarrellii Bourcier, 1847; Thaumastura cora Lesson \& Garnot, 1827; Rhodopis vesper Lesson, 1829 y Xenospingus concolor D’Orbignyi \& Lafresnaye, 1837 (CRACRAFT, 1995).

Acerca de la vegetación urbana, la ciudad de Tacna está compuesta principalmente por especies exóticas y sus áreas verdes han sido estimadas en $3 \mathrm{~m}^{2}$ por habitante, unos de los valores más altos en el Perú (ZEGARRA, 1995; INEI, 2015). Además, el 90\% de la población de la región se concentra en la ciudad de Tacna, con un promedio de edad de 32,5 años, mientras que el $24 \%$ de la población tiene formación universitaria, manteniendo una rica tradición basada en la cultura Aymara a través de sus descendientes, las toponimias y el comercio de productos tradicionales (PIZARRO, 2013; INEI, 2018).

\subsubsection{Metodología}

Durante el dictado del curso de estudios generales de estudios de pregrado denominado Ecología y Ambiente en la Universidad Nacional Jorge Basadre (UNJBG), a cargo del autor se invitó a estudiantes de todas las facultades para participar en un estudio para explorar su conocimiento acerca de nombres de aves. La muestra seleccionada correspondió a 340 estudiantes que dieron su consentimiento verbal para participar en la investigación y que cursaban el primer año de las facultades de Ciencias Médicas; Ciencias; Ingeniería; Ciencias Jurídicas y Empresariales; Ciencias Agropecuarias; Ingeniería Civil, Arquitectura y Geotecnia; y de Educación, Comunicación y Humanidades. La muestra representó el 20\% de los estudiantes que ingresaron durante el año 2019 a realizar estudios de pregrado en la UNJBG.

La colecta de datos se realizó en los ambientes de la Universidad Nacional Jorge Basadre de Tacna durante los meses de octubre y noviembre del año 2019. Los participantes recibieron un formato para completar datos de edad, facultad y elaborar una lista de nombres de aves en un plazo de 10-15 minutos. El método empleado fue el de lista libre o Free-listing, siguiendo a QUINLAN (2019). Para lograr hallar los nombres de aves con mayor relevancia se empleó el índice de importancia relativa (IR) que es una adaptación del índice de dominancia empleado por PIZARRO (2011) para evaluar listas de nombres de la fauna marina. El índice IR se calcula de la siguiente forma:

$$
\mathrm{IR}=\Sigma \mathrm{L} / \mathrm{N}
$$

Donde $\Sigma \mathrm{L}=$ es la sumatoria de todas las listas que contienen el nombre de 
Pizarro NEYRA, J. Predominancia ecológica y taxonómica de nombres de aves entre estudiantes universitarios peruanos. Ethnoscientia v. 6, n. 1, 2021. DOI: 10.22276/ethnoscientia.v6i1.353

determinada ave y $\mathrm{N}$ representa el número total de listas, en este caso $\mathrm{N}=340$.

Mediante el cálculo del índice IR se pudo establecer el peso que cada nombre de ave tenía en todas las listas obtenidas de los participantes. Se cotejaron los nombres de aves que aparecen en las listas con las especies de aves con las que se asocian (ver Apéndice 1). Adicionalmente se estimaron valores promedio de nombres de aves por persona.

A continuación se estimó el valor de importancia ecológica o ecological salience (ES) para todos los nombres de aves mencionados. Se tomaron en cuenta la información de distribución geográfica de las especies mencionadas en la literatura (PIZARRO, 1995; HØGSÅS; PIZARRO, 1997; SCHULENBERG et al., 2007; PIZARRO, 2020). En este caso se ordenaron los valores del índice IR en tres grupos: aves de distribución local (L), presentes en la ciudad de Tacna; aves introducidas, domésticas, asilvestradas y/o manejadas (M) en la ciudad de Tacna y aves ausentes (A) en la ciudad de Tacna. Luego se obtuvo el índice ES de la siguiente forma:

$$
\text { Índice } \mathrm{ES}=\left[\left(\sum \mathrm{L}+\sum \mathrm{M}\right)-\sum \mathrm{A}\right] /\left(\sum \mathrm{L}+\sum \mathrm{M}+\sum \mathrm{A}\right)
$$

Donde: $\Sigma \mathrm{L}=$ sumatoria de los valores IR de nombres de aves locales; $\Sigma \mathrm{M}=$ sumatoria de valores IR de nombres de aves manejadas/asilvestradas y $\Sigma \mathrm{A}=$ sumatoria de valores IR de nombres de aves ausentes en la ciudad de Tacna. El valor puede oscilar entre -1 y +1 , siendo negativo si predominan nombres de aves ausentes o sin predominancia ecológica, mientras valores $>0$ indica una predominancia de nombres de aves que se distribuyen en la zona o que han sido introducidas. Éste índice utiliza el valor del índice de importancia relativa (IR) de cada nombre de ave para que el resultado no solo esté basado en la distribución geográfica y en valores de ausencia/presencia, sino que refleje el consenso de todas las listas.

Finalmente, se estimó el índice de importancia taxonómica o taxonomic salience (TS), para lo cual se emplearon nuevamente los valores del índice IR para cada nombre. Primeramente se agruparon los nombres por orden taxonómico siguiendo a REMSEN et al., 2020 y, a continuación se sumaron los valores de IR dentro de cada orden y se obtuvo la proporción de importancia relativa de cada orden según:

$$
\mathrm{TS}(\text { Orden })=\left[\left(\sum \mathrm{EO}\right) /\left(\sum \mathrm{T}\right)^{2}\right] \mathrm{X} 100
$$

Donde: $\Sigma$ EO representa la sumatoria de valores IR de nombres de aves pertenecientes 
Pizarro NEYRA, J. Predominancia ecológica y taxonómica de nombres de aves entre estudiantes universitarios peruanos. Ethnoscientia v. 6, n. 1, 2021. DOI: 10.22276/ethnoscientia.v6i1.353

a determinado orden, mientras $\Sigma \mathrm{T}$ es equivalente a la suma total de valores IR de todos los nombres de aves colectados. El índice varía de 0 a 1 y emplea también valores del índice IR para darle un mayor peso a los nombres de aves más mencionados en las listas.

\section{Resultados y Discusión}

De acuerdo a los resultados, los estudiantes de primer año de la Universidad Nacional Jorge Basadre de Tacna escribieron en las listas un promedio de 14.76 nombres de aves por lista. Este es un valor considerablemente mayor que el obtenido con niños de la costa peruana en una investigación anterior (PIZARRO, 2011). Teniendo en cuenta que la cantidad de especies de aves que habitan el valle de Tacna fue estimada en 57 especies (HØGSÅS; PIZARRO, 1997), entonces el promedio obtenido de aves escrita por persona es exiguo si lo comparamos con resultados de investigaciones similares realizadas en países occidentales. Por ejemplo, Clergueau et al. (2011) encontraron que los pobladores urbanos de Rennes, Francia podían identificar once de las 17 especies de aves censadas en su zona.

En este trabajo, los estudiantes escribieron un total de 5535 nombres de aves correspondientes a 185 diferentes nombres, que a su vez se pueden identificar como etnoespecies. Durante la revisión de las listas se hallaron menciones a seres vivos extintos como el "Dodo", mamíferos como murciélago, ornitorrinco y roedores. También se encontraron los nombres "Fénix", un personaje mitológico, y "Charizard", un personaje de un anime de televisión. En el procesamiento de datos de las listas se eliminaron varios nombres de aves para evitar tomar en cuenta datos reiterativos y no relevantes como menciones de bromas o apodos a compañeros, así como menciones de nombres como "pájaro", "pajarito", "ave grande", "ave” y nombres similares, que no fueron contabilizados por carecer de valor como nombre a nivel de género o etnoespecie.

Los nombres fueron listados en orden descendente usando el valor del índice de importancia relativa (IR) y acompañados de la cita de la literatura que valida la correspondencia con alguna especie de ave. En caso contrario se considera que el nombre del ave es imposible de correlacionarlo con algún tipo de ave. (Apéndice 1).

Por otro lado, murciélagos, roedores y ornitorrincos fueron mencionados en las listas de nombres comunes de aves. En algunas culturas tradicionales los murciélagos pueden ser un tipo especial de aves, como sucede con el pueblo Karam de Nueva Guinea, la etnia Nage de Indonesia, o los Shipibo-Konibo de la Amazonía de Perú (BULMER, 1967; TOURNON, 1994; FORTH, 2010). Mientras que los murciélagos pueden ser clasificados como aves por adolescentes de sociedades occidentales, en algunas zonas urbanas de Brasil los murciélagos 
pueden ser confundidos con roedores o aves (TARKOWSKI, 2011; GOMES; COSTA NETO; ALVAREZ, 2017). En el caso de la presencia del término “ornitorrinco" en las listas, puede explicarse porque algunos de los estudiantes recurren a la similitud por percepción para clasificar objetos y seres vivos (LANDAU; SMITH; JONES, 1998). Ya que las aves y el ornitorrinco presentan un pico, ello podría inducir a algunos alumnos a clasificar a los dos seres vivos en base a su forma y no tomando en cuenta la similitud profunda que implica conocer conceptos acerca de la naturaleza de cada especie.

\subsection{Predominancia Ecológica}

Los resultados del índice de predominancia ecológica (ES) revelan que este es equivalente a 0.06. Es decir, es muy bajo e indica apenas una pequeña predominancia de nombres de especies locales en las listas frente a nombres de especies exóticas. (Ver Tabla $1)$.

\begin{tabular}{|l|c|c|c|c|}
\hline \multicolumn{5}{|c|}{ Tabla 1: Resultados del Índice de Predominancia Ecológica } \\
\hline Variables & $\sum \mathrm{L}$ & $\sum \mathrm{M}$ & $\sum \mathrm{A}$ & Índice ES \\
\hline Todas las listas & 5.29 & 4.58 & 8.7 & 0.06 \\
\hline
\end{tabular}

Fuente: el autor (2020).

Estos resultados pueden explicarse por la falta de experiencia directa con animales silvestres en habitantes urbanos, la cual es suplida por información proveniente de medios de comunicación y de material escrito, trayendo como consecuencia una mayor familiaridad con especies exóticas que locales (WINKLER-RHOADES et al., 2010). Sin duda es un tema que requiere mayor investigación, ya que la recuperación de la información de dominios semánticos no solo esta mediada culturalmente sino también por emociones y sentimientos ligados a los seres vivos (NOLAN et al., 2006). En un trabajo similar realizado en la región (PIZARRO, 2011), se consideró que la migración reciente, que es muy intensa en esta zona, puede traer como consecuencia una falta de saberes previos sobre la fauna local debido a que las especies que se distribuyen en su nuevo lugar de residencia no se encuentran contenidas en el capital cultural de los inmigrantes, trayendo como consecuencia una falta de transmisión de conocimientos locales a los hijos. Lo anterior podría haber afectado también a los estudiantes con padres migrantes.

\subsection{Predominancia Taxonómica}

Los resultados muestran una predominancia taxonómica de órdenes con mucha 
riqueza de especies, así como de importancia económica y cultural. Los órdenes de la SubClase Neornithes con un índice TS $>0.4$ son: Galliformes, Passeriformes, Anseriformes, Psittaciformes, Cathartiformes y Rheiformes. (Ver Figura 1).

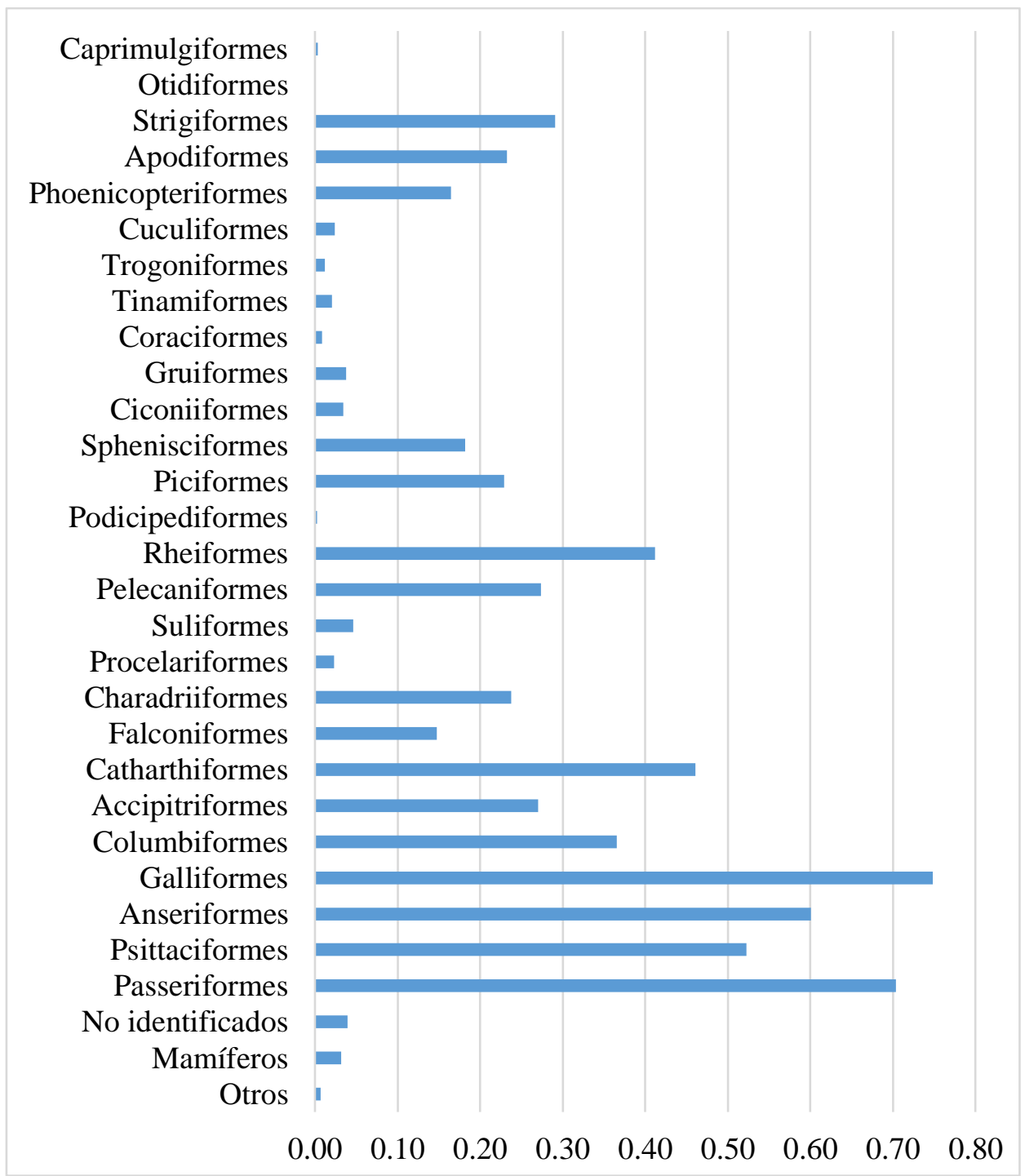

Figura 1: Predominancia Taxonómica de nombres de Aves por Orden según Estudiantes Universitarios Peruanos. Fuente: el autor (2020).

Estos resultados son previsibles, ya que la carne de aves como las Galliformes y Anseriformes ocupa el segundo lugar en el consumo humano de carnes en el mundo después de la de cerdo (HENCHION et al., 2014). Un hecho que puede explicar esta predominancia taxonómica es el crecimiento en el consumo de Galliformes que se ha experimentado en el Perú durante los últimos cuarenta años que, aunado al boom gastronómico reciente, ha promovido el aumento de su consumo (JAKOBY, 2012). Además, la crisis económica ha devenido en actividades de colecta de fondos a través de las llamadas "polladas" (BÉJAR; 
Pizarro NEYRA, J. Predominancia ecológica y taxonómica de nombres de aves entre estudiantes universitarios peruanos. Ethnoscientia v. 6, n. 1, 2021. DOI: 10.22276/ethnoscientia.v6i1.353

ÁLVAREZ ALDERETE, 2010).

Los Anseriformes también tienen una alta predominancia por las mismas razones en el Perú (CABIESES, 1995; ZAPATA, 2006; ANTÚNEZ DE MAYOLO, 2011). No es sorprendente tampoco que los Passeriformes aparezcan como el orden con el segundo valor más alto del índice de predominancia taxonómica, debido a la diversidad de especies y familias que tiene. Además, se ha encontrado que los Passeriformes son el grupo preferido de aves entre adolescentes (BJERKE; ØSTDAHL, 2004). En el caso de los Psittaciformes, se trata de un grupo formado por aves carismáticas preferidas como mascotas en todo el mundo debido a su capacidad de imitar voces humanas, su inteligencia, belleza y docilidad (NOBREGA ALVES; ALVES ROCHA, 2018). Su presencia en las ciudades peruanas se debe a aves escapadas que se han asilvestrado y ello se ha acentuado debido al intenso tráfico ilegal de estas especies en Perú (GASTAÑAGA et al., 2011). En tanto las Cathartiformes, serían sobresalientes taxonómicamente gracias al alto valor de importancia relativa alcanzado por Vultur gryphus Linnaeus "Cóndor andino" y Cathartes aura (Molina) "Gallinazo". En especial el Cóndor andino está presente en la cultura popular a través de apellidos, rituales, cuentos y toponimias (VENERO, 2008; FRANCO INOJOSA, 2017; ROJAS, 2018; PIZARRO, 2020). Finalmente, las Rheiformes pueden haber alcanzado popularidad entre los estudiantes ya que la especie Rhea pennata D’Orbigny, está amenazada en el Perú y es muy conocida en los últimos años debido a que en la región se ha creado un área protegida para conservar a la especie (SERFOR, 2018).

La explicación más plausible para una predominancia de especies exóticas y solo unos pocos órdenes sobresalientes taxonómicamente puede estar en la dificultad para la observación de las aves locales, hecho que ya mencionó Hunn (1999) para aves como las rapaces nocturnas. Berlin (1992) también menciona que las respuestas sobre nomenclatura de aves requieren además que los participantes conozcan el plumaje y el tamaño del ave, hecho que es muy difícil que ocurra entre pobladores urbanos.

\section{CONCLUSIONES}

Después de emplear el índice propuesto para medir Predominancia ecológica, se puede afirmar que los estudiantes de la Universidad Nacional Jorge Basadre han escrito en las listas un mayor número de nombres de aves introducidas y ausentes del hábitat urbano en comparación con los nombres de aves locales, aunque algunas sobresalen también en las listas por ser aves emblemáticas, como Vultur gryphus "Cóndor andino", Rupicola peruviana “Gallito de las rocas”, Struthio camelus "Avestruz", Pelecanus thagus "Pelícano peruano", 
Spheniscus humboldtii "Pingüino de Humboldt", entre otros.

En el caso del índice de Predominancia Taxonómica, los órdenes que exhiben una mayor importancia relativa son aquellos que igualmente reúnen especies de valor económico y recreativo como Galliformes, Anseriformes y Psittaciformes, asi como otras aves muy comunes en la ciudad como loros, gallinazos y Passeriformes como los gorriones, aunque en casi todos los órdenes se observa que abundan los nombres de especies exóticas.

\section{AGRADECIMIENTOS}

Se agradece la colaboración de los estudiantes de la Universidad Nacional Jorge Basadre de Tacna quienes accedieron voluntariamente a participar de forma anónima en el presente trabajo de investigación, así como a los árbitros y al editor de Ethnoscientia, quienes aportaron mucho para mejorar el texto. Agradezco a la Asociación para el Desarrollo de las Ciencias Biológicas por haber otorgado patrocinio a la presente investigación.

\section{REFERENCIAS BIBLIOGRÁFICAS}

ANTÚNEZ DE MAYOLO, S. E. La Nutrición en el Antiguo Perú. Sexta edición. Lima: Sociedad Geográfica de Lima, 2011. 240 p.

BÉJAR RIVERA, H.; ÁLVAREZ ALDERETE, M. A. Las polladas: una estrategia de sobrevivencia en época de crisis económica y política. Lima, 1980-2003. Investigaciones sociales 14(24): 259-283, 2010.

BERLIN, B. Ethnobiological classification: Principles of categorization of plants and animals in traditional societies. New Jersey: Princeton University Press, 1992. 336 p.

BIRDLIFE INTERNATIONAL (2020). Country profile: Peru. Disponible en: http://www.birdlife.org/datazone/country/peru. Verificado en: 23 jun. 2020.

BJERKE, T.; ØSTDAHL, T. Animal-related attitudes and activities in an urban population. Anthrozoös 17(2): 109-129, 2004.

BULMER, R. Why is the Cassowary Not a Bird? A Problem of Zoological Taxonomy Among the Karam of the New Guinea Highlands. Man, New Series. 2(1): 5-25, 1967.

CABIESES, F. Cien Siglos de Pan: 10,000 años de alimentación en el Perú. Lima: A \& B Editores-Consejo Nacional de Ciencia y Tecnología, 1995.

CLERGUEAU P. et al. Human perception and appreciation of birds: A motivation for wildlife conservation in urban environments of France. En: J. M. Marzluff et al. (eds.), Avian Ecology and Conservation in an Urbanizing World. New York, Springer Science+Business Media, New York, 2001. 
CRACRAFT J. Historical Biogeography and Patterns of Differentiation within the South American Avifauna: Areas of Endemism. Ornithological Monographs, 36: 49-84, 1985.

DOUGHERTY, J. W. Salience and Relativity in Classification. American Ethnologist 5(1): 66-80, 1978.

FARIAS, G. B.; ALVES, A. G. C. É importante pesquisar o nome local das aves? Revista Brasileira de Ornitologia, 15(3): p. 403-408, 2007.

FORTH, G. Symbolic and ironic bats: varieties of Classification in Nage Folk Ornithology. Ethnology, 48(2): 139-159, 2010.

FRANCO INOJOSA, M., Fábulas Orales Aimaras. Lima: Ministerio de Educación. Dirección General de Educación Básica Alternativa, Intercultural y de Servicios Educativos en el Ámbito Rural, 2017. 82 p.

GASTAÑAGA, M. et al. A study of the parrot trade in Peru and the potential importance of internal trade for threatened species. Bird Conservation International 21(1): 76-85, 2011.

GOMES, M. D. C. B.; COSTA NETO, E. M.; ALVAREZ, M. E. D. V. Ethnozoology of bats (Mammalia, Chiroptera) in Feira de Santana Municipality, Bahia State, Northeastern Brazil. Brazilian Journal of Biological Sciences 4(7): 147-156, 2017.

HENCHION, M. et al. Meat consumption: Trends and quality matters. Meat science 98(3): 561-568, 2014.

HØGSÅS, T. E.; PIZARRO, J. Algunas plantas visitadas por Aves en Tacna. Libro de Resúmenes de II Jornada Nacional Ornitológica. Arequipa: Grupo de Aves del Perú. 1997.

HUNN, E. Size as limiting the recognition of biodiversity in folkbiological classifications: One of four factors governing the cultural recognition of biological taxa. In: Medin, D. y S. Atran (eds.). Folkbiology. Massachusetts: Bradford Book/MIT Press. Chapter 3. 47-69, 1999.

INEI. Registro Nacional de Municipalidades 2014. Principales Resultados. Lima: Instituto Nacional de Estadística e Informática, 2015.

INEI. Perfil Sociodemográfico del Perú, Informe Nacional Censos Nacionales XII de Población, VII de Vivienda y III de Comunidades Indígenas. Lima: Instituto Nacional de Estadística e Informática, 2018.

JAKOBY, E. Come rico, come sano, come peruano... y empecemos a trabajar en eso. Revista Instituto Nacional de Salud 18(7-8):163-168, 2012.

LANDAU, B.; SMITH, L.B.; JONES, S. S. The Importance of shape in Early Lexical Learning. Cognitive Development 3: 299-321, 1988.

NOBREGA ALVES, R. R.; ALVES ROCHA, L. Fauna at Home: Animals as Pets. In: Nobrega, R. R. \& U.P. Albuquerque, (eds.). Ethnozoology, Animals in Our Lives. London: Academic Press/Elsevier Inc, 2018. Chapter 16. 303-322 p. 
NOLAN, J. M. et al. The lovable, the loathsome, and the liminal: emotionality in ethnozoological cognition. Journal of Ethnobiology, 26(1): 126-138, 2006.

PERÚ. Ministerio del Ambiente. V Informe Nacional sobre la Aplicación del Convenio sobre la Diversidad Biológica. (2010-2013). Lima: Comisión Nacional de Diversidad Biológica CONADIB-Grupo Técnico Ad-Hoc del V Informe Nacional, 2018.

PIZARRO, J. Observaciones de Avifauna Nativa de Tacna, Resultados Preliminares. Ciencia \& Desarrollo-UNJBG (1): 20-25, 1995.

PIZARRO, J. Peruvian Children's Folk Taxonomy of Marine Animals. Ethnobiology Letters (2): 50-57, 2011.

PIZARRO, J. Formas de vida en la Etnobotánica Aymara. Indiana 30: 301-323, 2013.

PIZARRO, J. Diccionario de las Aves de Tacna. Tacna: Asociación para el Desarrollo de las Ciencias Biológicas en el Perú, Tacna. 2020. 75 p.

QUINLAN, M.B. The Freelisting Method. In: P. Liamputtong (ed.), Handbook of Research Methods in Health Social Sciences. Singapore: Springer Nature Singapore Pte Ltd. Chapter 82. 1431-1446, 2019.

REMSEN, J. V. et al. Version [21 July 2020]. A classification of the bird species of South America. American Ornithological Society. Disponible en http://www.museum.lsu.edu/ Remsen/SACCBaseline.htm. Verificado en: 20 de jul de 2020.

ROJAS, M. La diversidad en los antropónimos de los peruanos. Desde el Sur 10(2): 331$345,2018$.

SAlinAS L.; ABARCA, M.; WUST, W. Aves del Perú. Lima: Grupo La República Publicaciones S.A., 2018. 120 p.

SERFOR. Libro Rojo de la Fauna Silvestre Amenazada del Perú. Primera edición. Lima: Servicio Nacional Forestal y de Fauna Silvestre, 2018. 528p.

SCHUlENBERG, T. S. et al. Birds of Peru. Princeton, New Jersey: Princeton University Press, 2007. 656 p.

WINKLER-RHOADES, N. et al. Naming the animals that come to mind: Effects of culture and experience on category fluency. Journal of Cognition and Culture 10(1-2): 205-220, 2010.

TARLOWSKI, A. Naming patterns and inductive inference: the case of birds. Journal of Cognition and Culture 11(1-2): 189-216, 2011.

TOURNON, J. Cómo los Shipibo-Konibo Nombran y Clasifican los Animales. Antropológica (Lima) 11: 91-108, 1994.

VENERO, J.L. 2008. Etnoornitología y Guía de Aves del Humedal "Lucre-Huacarpay". 
Pizarro NEYRA, J. Predominancia ecológica y taxonómica de nombres de aves entre estudiantes universitarios peruanos. Ethnoscientia v. 6, n. 1, 2021. DOI: 10.22276/ethnoscientia.v6i1.353

Cusco: Editorial Moderna, 2008. 250 p.

ZAPATA, S. Diccionario de Gastronomía Peruana Tradicional. Lima: Universidad de San Martín de Porres, 2006.736 p.

ZEGARRA, R. Plantas Ornamentales de la Ciudad de Tacna. Ciencia \& Desarrollo-UNJBG (2): 23-31, 1995. 


\section{APÉNDICE 1}

Nombres populares de las Aves ordenados según Índice de Importancia Relativa.

\section{APPENDIX 1}

Folk Bird Names according with Relative Importance Index

\begin{tabular}{|c|c|c|c|c|}
\hline $\begin{array}{l}\text { FOLK NAME- } \\
\text { DISTRIBUTION/ } \\
\text { NOMBRE } \\
\text { COMÚN- } \\
\text { DISTRIBUCIÓN } \\
*\end{array}$ & $\begin{array}{l}\text { ORDER/ } \\
\text { ORDEN }\end{array}$ & $\begin{array}{l}\text { CORRESPONDING } \\
\text { SPECIES/ } \\
\text { ESPECIE }\end{array}$ & $\mathbf{n}$ & $\begin{array}{l}\text { IR } \\
\text { Index }\end{array}$ \\
\hline Gallina $(\mathrm{M})$ & GALLIFORMES & Gallus gallus (Linnaeus, 1758) & 297 & 0.8735 \\
\hline Paloma (L), (M) & COLUMBIFORMES & $\begin{array}{l}\text { Columba livia (Gmelin, 1789); } \\
\text { Zenaida meloda (Tschudi 1843); } \\
\text { Columbina cruziana (Prévost, } \\
\text { 1842); Zenaida auriculata, (Des } \\
\text { Murs, 1847) }\end{array}$ & 279 & 0.8206 \\
\hline Pato (M) & ANSERIFORMES & $\begin{array}{l}\text { Anas platyrrhynchus (Linnaeus, } \\
\text { 1758); Cairina moschata } \\
\text { (Linnaeus, } 1758 \text { ) }\end{array}$ & 274 & 0.8059 \\
\hline Cóndor (A) & CATHARTIFORMES & Vultur gryphus (Linnaeus, 1758) & 216 & 0.6353 \\
\hline Loro $(\mathrm{M}),(\mathrm{L})$ & PSITTACIFORMES & $\begin{array}{l}\text { Psittacara mitratus } \text { (Tschudi, } \\
\text { 1844); Psittacara wagleri (Gray, } \\
\text { 1845); Psittacara erythrogenys } \\
\text { (Lesson, 1844); Amazona } \\
\text { amazónica (Linnaeus, 1766); } \\
\text { Amazona aestiva (Linnaeus, } \\
\text { 1758); Psilopsiagon aurifrons } \\
\text { (Lesson, 1830); Brotogeris } \\
\text { versicolorus (Muller, 1776) }\end{array}$ & 212 & 0.6235 \\
\hline Picaflor, colibri (L) & APODIFORMES & $\begin{array}{l}\text { Thaumastura cora (Lesson; } \\
\text { Garnot, 1827); Rhodopis vesper } \\
\text { (Lesson, 1829); Eulidia yarrellii } \\
\text { (Bourcier, 1847) y otros }\end{array}$ & 208 & 0.6118 \\
\hline Pavo (M) & GALLIFORMES & $\begin{array}{l}\text { Meleagris gallopavo (Linnaeus, } \\
1758 \text { ) }\end{array}$ & 198 & 0.5824 \\
\hline Gallito Rocas (A) & PASSERIFORMES & $\begin{array}{l}\text { Rupicola peruviana (Latham, } \\
1790 \text { ) }\end{array}$ & 182 & 0.5353 \\
\hline Aguila (A) & ACCIPITRIFORMES & $\begin{array}{ll}\begin{array}{l}\text { Geranoaetus } \\
\text { (Vieillot, 1819) }\end{array} & \text { melanoleucus } \\
\end{array}$ & 177 & 0.5206 \\
\hline Avestruz (A) & RHEIFORMES & $\begin{array}{l}\text { Struthio camelus (Linnaeus, } \\
1758)\end{array}$ & 175 & 0.5147 \\
\hline Pelícano (A) & PELECANIFORMES & Pelecanus thagus (Molina, 1782) & 172 & 0.5059 \\
\hline Gallinazo (L) & CATHARTIFORMES & $\begin{array}{l}\text { Catharthes aura (Linnaeus, } \\
1748 \text { ) }\end{array}$ & 152 & 0.4471 \\
\hline $\begin{array}{l}\text { Guacamayo, } \\
\text { Papagallo (M) }\end{array}$ & PSITTACIFORMES & Ara spp. (Lacépède, 1799) & 148 & 0.4353 \\
\hline Pingüino (A) & SPHENISCIFORMES & $\begin{array}{l}\text { Spheniscus humboldti (Meyen, } \\
\text { 1834) y otros penguins }\end{array}$ & 147 & 0.4324 \\
\hline Tucán (A) & PICIFORMES & $\begin{array}{lll}\begin{array}{l}\text { Rhampastos } \\
\text { (Muller, 1785) }\end{array} & \text { toco } & \text { Statius } \\
\end{array}$ & 145 & 0.4265 \\
\hline
\end{tabular}


Pizarro NEYRA, J. Predominancia ecológica y taxonómica de nombres de aves entre estudiantes universitarios peruanos. Ethnoscientia v. 6, n. 1, 2021. DOI: 10.22276/ethnoscientia.v6i1.353

\begin{tabular}{|c|c|c|c|c|}
\hline Ganso (M) & ANSERIFORMES & Anser anser (Linnaeus, 1758) & 144 & 0.4235 \\
\hline Buho (L) & STRIGIFORMES & $\begin{array}{l}\text { Bubo virginianus (Gmelin, } \\
1788) \text {; Tyto alba (Scopoli, 1769) }\end{array}$ & 144 & 0.4235 \\
\hline Gaviota (A) & CHARADRIIFORMES & $\begin{array}{l}\text { At least Larus dominicanus } \\
\text { (Lichtenstein, 1823): Larus } \\
\text { belcheri Vigors, 1829; Larus } \\
\text { modestus (Tschudi, 1843) }\end{array}$ & 136 & 0.4000 \\
\hline Lechuza (L) & STRIGIFORMES & $\begin{array}{l}\text { Athene cunicularia (Molina, } \\
\text { 1782) }\end{array}$ & 109 & 0.3206 \\
\hline Halcón (L) & FALCONIFORMES & $\begin{array}{l}\text { Falco femoralis } \quad \text { (Temminck, } \\
\text { 1822); Falco } r \text { sparverius } \\
\text { (Linnaeus, 1758); F. peregrinus } \\
\text { (Tunstall. 1771) }\end{array}$ & 108 & 0.3176 \\
\hline Ñandú (A) & RHEIFORMES & $\begin{array}{l}\text { Rhea americana (Linnaeus, } \\
1758)\end{array}$ & 99 & 0.2912 \\
\hline Codorniz (M) & GALLIFORMES & $\begin{array}{l}\text { Coturnix coturnix (Linnaeus, } \\
1758 \text { ) }\end{array}$ & 92 & 0.2706 \\
\hline Cisne (A) & ANSERIFORMES & Cygnus spp. (Garsault, 1764) & 91 & 0.2676 \\
\hline Flamenco $(\mathrm{A})$ & $\begin{array}{l}\text { PHOENICOPTERIFO } \\
\text { RMES }\end{array}$ & $\begin{array}{l}\text { Phoenicopteridae (Bonaparte, } \\
\text { 1831) }\end{array}$ & 80 & 0.2353 \\
\hline Suri (A) & RHEIFORMES & Rhea pennata (d'Orbingy, 1834) & 79 & 0.2324 \\
\hline Canario (M) & PASSERIFORMES & $\begin{array}{l}\text { Aves Paseriformes de diferentes } \\
\text { especies }\end{array}$ & 76 & 0.2235 \\
\hline Gorrión (L) & PASSERIFORMES & $\begin{array}{l}\text { Passer domesticus (Linnaeus, } \\
\text { 1758) and Zonotrichia capensis } \\
\text { (Statius Muller, 1776) }\end{array}$ & 73 & 0.2147 \\
\hline Parihuana (A) & $\begin{array}{l}\text { PHOENICOPTERIFOR } \\
\text { MES }\end{array}$ & $\begin{array}{ll}\begin{array}{l}\text { Phoenicopterus } \\
\text { (Molina, 1782) }\end{array} & \text { chilensis } \\
\end{array}$ & 69 & 0.2029 \\
\hline Cuervo (A) & PASSERIFORMES & Corvus spp. (Linnaeus, 1758) & 67 & 0.1971 \\
\hline Pavo real $(\mathrm{M})$ & PSITTACIFORMES & Pavo cristatus (Linnaeus, 1758) & 66 & 0.1941 \\
\hline $\begin{array}{l}\text { Pajaro carpintero } \\
\text { (A) }\end{array}$ & PICIFORMES & $\begin{array}{l}\text { Colaptes atricollis (Malherbe, } \\
\text { 1850) }\end{array}$ & 62 & 0.1824 \\
\hline Golondrina (L) & PASSERIFORMES & $\begin{array}{ll}\begin{array}{l}\text { Pygochelidon } \\
\text { (Vieillot, 1817) }\end{array} & \text { cyanoleuca } \\
\end{array}$ & 60 & 0.1765 \\
\hline Buitre (A) & ACCIPITRIFORMES & Gyps fulvus (Hablizi, 1783) & 48 & 0.1412 \\
\hline Garza (L) & PELECANIFORMES & $\begin{array}{l}\text { Bubulcus ibis (Linnaeus, 1758); } \\
\text { Egretta thula (Molina, 1782) }\end{array}$ & 47 & 0.1382 \\
\hline Perico $(\mathrm{L})$ & PSITTACIFORMES & $\begin{array}{l}\text { Psilopsiagon aurifrons (Lesson, } \\
1830 \text { ) }\end{array}$ & 39 & 0.1147 \\
\hline Gavilán (L) & ACCIPITRIFORMES & Circus cinereus (Vieillot, 1816) & 33 & 0.0971 \\
\hline Cigueña (A) & CICONIIFORMES & Ciconia sp. (Brisson, 1760) & 31 & 0.0912 \\
\hline Petirrojo (L) & PASSERIFORMES & $\begin{array}{l}\text { Pyrocephalus rubinus (Boddaert, } \\
1783 \text { ) }\end{array}$ & 28 & 0.0824 \\
\hline Urraca (A) & PASSERIFORMES & $\begin{array}{ll}\text { Cyanocorax } & \text { mystacalis } \\
\text { (Geoffroy Saint-Hilaire, } 1835 \text { ) }\end{array}$ & 25 & 0.0735 \\
\hline Cacatua $(\mathrm{A})$ & PSITTACIFORMES & Cacatuidae (Gray, 1840) & 23 & 0.0676 \\
\hline Cernicalo (L) & FALCONIFORMES & $\begin{array}{lll}\text { Falco sparverius } & \text { (Linnaeus, } \\
1758)\end{array}$ & 23 & 0.0676 \\
\hline Ostrero (A) & CHARADRIIFORMES & $\begin{array}{ll}\begin{array}{l}\text { Haematopus } \\
\text { (Temminck, 1820) }\end{array} & \text { palliatus } \\
\end{array}$ & 23 & 0.0676 \\
\hline Grulla (A) & GRUIFORMES & Grus sp. (Brisson, 1760) & 22 & 0.0647 \\
\hline Zorzal (A) & PASSERIFORMES & Turdus chiguanco (Lafresnaye; & 22 & 0.0647 \\
\hline
\end{tabular}


Pizarro NEYRA, J. Predominancia ecológica y taxonómica de nombres de aves entre estudiantes universitarios peruanos. Ethnoscientia v. 6, n. 1, 2021. DOI: 10.22276/ethnoscientia.v6i1.353

\begin{tabular}{|c|c|c|c|c|}
\hline & & D’Orbigny, 1837) & & \\
\hline Cuculi (L) & COLUMBIFORMES & Zenaida meloda (Tschudi, 1843) & 19 & 0.0559 \\
\hline Pato salvaje (A) & ANSERIFORMES & Anatidae (Leach, 1820) & 19 & 0.0559 \\
\hline Patillo (A) & SULIFORMES & $\begin{array}{l}\text { Phalacrocorax brasilianus } \\
\text { (Gmelin, 1789); Phalacrocorax } \\
\text { bougainvilllii (Lesson, 1837) }\end{array}$ & 18 & 0.0529 \\
\hline Alcatraz (A) & PELECANIFORMES & Pelecanus thagus (Molina, 1782) & 18 & 0.0529 \\
\hline Kiwi (A) & RHEIFORMES & Apteryx australis (Shaw, 1813) & 17 & 0.0500 \\
\hline Perdiz (A) & TINAMIFORMES & $\begin{array}{l}\text { Nothoprocta ornata (Gray, } \\
\text { 1867); Nothoprocta pentlandi } \\
\text { (Gray, 1867) }\end{array}$ & 17 & 0.0500 \\
\hline Murcielago (L) & MAMMALIA & $\begin{array}{l}\text { Chiroptera (Blumenbach, 1779) } \\
\text { (Mammalia) }\end{array}$ & 15 & 0.0441 \\
\hline Pava aliblanca $(\mathrm{A})$ & GALLIFORMES & $\begin{array}{l}\text { Penelope } \\
\text { (Taczanowski, 1878) }\end{array}$ & 15 & 0.0441 \\
\hline Albatros (A) & PROCELLARIFORMES & Diomedeidae (Gray 1840) & 14 & 0.0412 \\
\hline Jilguero (L) & PASSERIFORMES & $\begin{array}{l}\text { Spinus magellanicus (Vieillot, } \\
\text { 1805) }\end{array}$ & 14 & 0.0412 \\
\hline Piquero (A) & SULIFORMES & Sula variegata (Tschudi, 1843) & 14 & 0.0412 \\
\hline Loro azulejo (A) & PSITTACIFORMES & Psittaciforme no identificado & 14 & 0.0412 \\
\hline Gaviota reidora $(\mathrm{A})$ & CHARADRIIFORMES & $\begin{array}{ll}\text { Chroicocephalus } & \text { ridibundus } \\
\text { (Linnaeus, 1766) } & \end{array}$ & 14 & 0.0412 \\
\hline Tortola (L) & COLUMBIFORMES & $\begin{array}{l}\text { Columbina cruziana (Prévost, } \\
\text { 1842) }\end{array}$ & 13 & 0.0382 \\
\hline Leque leque $(\mathrm{L})$ & CHARADRIIFORMES & $\begin{array}{l}\text { Burhinus superciliaris (Tschudi, } \\
\text { 1843) }\end{array}$ & 13 & 0.0382 \\
\hline $\begin{array}{l}\text { Pingüino Humboldt } \\
\text { (A) }\end{array}$ & SPHENISCIFORMES & $\begin{array}{l}\text { Spheniscus humboldti (Meyen, } \\
\text { 1834) }\end{array}$ & 12 & 0.0353 \\
\hline Dodo $(\mathrm{A})$ & COLUMBIFORMES & $\begin{array}{l}\text { Rhaphus cucullatus (Linnaeus, } \\
\text { 1758). }\end{array}$ & 11 & 0.0324 \\
\hline Pichuncho (L) & PASSERIFORMES & $\begin{array}{l}\text { Zonotrichia capensis (Statius } \\
\text { Muller, 1776) }\end{array}$ & 11 & 0.0324 \\
\hline Quetzal (A) & TROGONIFORMES & $\begin{array}{l}\text { Pharomacrus mocinno (de La } \\
\text { Llave, 1832) }\end{array}$ & 10 & 0.0294 \\
\hline Pericote (L) & MAMMALIA & $\begin{array}{l}\text { Rodentia (Bowdich, 1821) } \\
\text { (Mammalia) }\end{array}$ & 10 & 0.0294 \\
\hline Huallata (A) & ANSERIFORMES & $\begin{array}{l}\text { Oressochen melanopterus } \\
\text { (Eyton, 1838) }\end{array}$ & 10 & 0.0294 \\
\hline Correcaminos (A) & CUCULIFORMES & $\begin{array}{ll}\text { Geococcyx } & \text { californianus } \\
\text { (Lesson, 1829) } & \\
\end{array}$ & 10 & 0.0294 \\
\hline Cardenal (A) & PASSERIFORMES & $\begin{array}{l}\text { Paroaria coronata (Miller, } \\
1776)\end{array}$ & 10 & 0.0294 \\
\hline Ruiseñor (L) & PASSERIFORMES & $\begin{array}{l}\text { Troglodytes aedon (Vieillot, } \\
\text { 1809) }\end{array}$ & 10 & 0.0294 \\
\hline Catita (L) & PSITTACIFORMES & $\begin{array}{l}\text { Psilopsiagon aurifrons (Lesson, } \\
\text { 1830) }\end{array}$ & 9 & 0.0265 \\
\hline $\begin{array}{l}\text { Quebrantahuesos } \\
\text { (A) }\end{array}$ & ACCIPITRIFORMES & $\begin{array}{l}\text { Gypaetus barbatus (Linnaeus, } \\
1758 \text { ) }\end{array}$ & 8 & 0.0235 \\
\hline Aguila Calva (A) & ACCIPITRIFORMES & $\begin{array}{l}\text { Haliaeetus leucocephalus } \\
\text { (Linnaeus, 1766) }\end{array}$ & 8 & 0.0235 \\
\hline $\begin{array}{l}\text { Martin pescador } \\
\text { (A) }\end{array}$ & CORACIFORMES & $\begin{array}{ll}\text { Chloroceryle } & \text { americana } \\
\text { (Gmelin, 1788) }\end{array}$ & 8 & 0.0235 \\
\hline
\end{tabular}


Pizarro NEYRA, J. Predominancia ecológica y taxonómica de nombres de aves entre estudiantes universitarios peruanos. Ethnoscientia v. 6, n. 1, 2021. DOI: 10.22276/ethnoscientia.v6i1.353

\begin{tabular}{|c|c|c|c|c|}
\hline Tordo (L) & PASSERIFORMES & $\begin{array}{l}\text { Molothrus bonariensis (Gmelin, } \\
\text { 1789) }\end{array}$ & 8 & 0.0235 \\
\hline Choca (A) & GRUIFORMES & Fulica ardesiaca (Tschudi, 1843) & 7 & 0.0206 \\
\hline $\operatorname{Cuco}(\mathrm{A})$ & CUCULIFORMES & $\begin{array}{l}\text { Tordo gigante Scaphidura } \\
\text { oryzivora (Gmelin, 1788) }\end{array}$ & 6 & 0.0176 \\
\hline Correlimos (A) & CHARADRIIFORMES & Calidris spp. (Merrem, 1804) & 6 & 0.0176 \\
\hline Chiguanco (A) & PASSERIFORMES & $\begin{array}{l}\text { Turdus chiguanco (Lafresnaye; } \\
\text { D’Orbigny, 1837) }\end{array}$ & 5 & 0.0147 \\
\hline Guanay (A) & SULIFORMES & $\begin{array}{l}\text { Phalacrocorax } \\
\text { (Lesson, 1837) }\end{array}$ & 5 & 0.0147 \\
\hline Guardacaballo (L) & CUCULIFORMES & $\begin{array}{ll}\text { Crotophaga } & \text { sulcirostris } \\
\text { (Swainson, 1827) } & \end{array}$ & 4 & 0.0118 \\
\hline Fenix (A) & $\begin{array}{l}\text { MYTHOLOGICAL } \\
\text { BIRD }\end{array}$ & & 4 & 0.0118 \\
\hline Chorlito (A) & CHARADRIIFORMES & $\begin{array}{l}\text { Scolopacidae } \text { (Rafinesque, } \\
\text { 1815); Charadriidae (Leach, } \\
\text { 1820) }\end{array}$ & 4 & 0.0118 \\
\hline Torcaza (L) & COLUMBIFORMES & $\begin{array}{l}\text { Palomas como } \text { Zenaida } \\
\text { auriculata (Des Murs, 1847) }\end{array}$ & 4 & 0.0118 \\
\hline Secretario (A) & ACCIPITRIFORMES & $\begin{array}{l}\text { Sagittarius serpentarius (Miller, } \\
1779 \text { ) }\end{array}$ & 4 & 0.0118 \\
\hline Faisan (A) & GALLIFORMES & $\begin{array}{l}\text { Phasianus colchicus (Linnaeus, } \\
\text { 1758) }\end{array}$ & 4 & 0.0118 \\
\hline Ornitorrinco (A) & $\begin{array}{l}\text { MAMMALIA } \\
\text { (MONOTREMATA) }\end{array}$ & $\begin{array}{l}\text { Ornithorhynchus anatinus } \\
\text { (Shaw, 1799) }\end{array}$ & 4 & 0.0118 \\
\hline Harpia (A) & ACCIPITRIFORMES & Harpia harpyia (Linnaeus, 1758) & 4 & 0.0118 \\
\hline Espatula (A) & PELECANIFORMES & Platalea ajaja (Linnaeus, 1758) & 4 & 0.0118 \\
\hline $\begin{array}{l}\text { Pingüino } \\
\text { Emperador }(\mathrm{A})\end{array}$ & SPHENISCIFORMES & $\begin{array}{l}\text { Aptenodytes forsteri (Gray, } \\
\text { 1844) }\end{array}$ & 4 & 0.0118 \\
\hline Ave del Paraiso (A) & PASSERIFORMES & Aves de la familia Paradisideae & 4 & 0.0118 \\
\hline Herrerillo (A) & PASSERIFORMES & Parus spp. (Linnaeus, 1758) & 4 & 0.0118 \\
\hline $\begin{array}{l}\text { Guacamayo azul } \\
\text { (A) }\end{array}$ & PSITTACIFORMES & Ara ararauna (Linnaeus, 1758) & 4 & 0.0118 \\
\hline $\begin{array}{l}\text { Guacamayo } \\
\text { Amarillo (A) }\end{array}$ & PSITTACIFORMES & Ara chloropterus (Gray, 1859) & 4 & 0.0118 \\
\hline $\begin{array}{l}\text { Guacamayo rojo } \\
\text { (A) }\end{array}$ & PSITTACIFORMES & Ara militaris (Linnaeus, 1766) & 4 & 0.0118 \\
\hline Aguilucho (L) & ACCIPITRIFORMES & $\begin{array}{l}\text { Geranoaetus polyosoma (Quoy; } \\
\text { Gaimard, 1824); Geranoaetus } \\
\text { melanoleucus (Vieillot, 1819) }\end{array}$ & 4 & 0.0118 \\
\hline Muttons (A) & PROCELLARIFORMES & $\begin{array}{l}\text { Puffinus } \\
\text { (Temminck, 1836) y onirostris } \\
\text { Procellariformes }\end{array}$ & 4 & 0.0118 \\
\hline Picozapato (A) & PELECANIFORMES & Balaeniceps rex (Gould, 1850) & 4 & 0.0118 \\
\hline Petizo (A) & DESCONOCIDO & & 4 & 0.0118 \\
\hline Ñusta (A) & DESCONOCIDO & & 4 & 0.0118 \\
\hline Carabo (A) & STRIGIFORMES & Strigidae (Leach, 1820) & 4 & 0.0118 \\
\hline Chortelito (A) & DESCONOCIDO & & 4 & 0.0118 \\
\hline Avechucho (A) & DESCONOCIDO & & 2 & 0.0059 \\
\hline Zambullidor (A) & PODICIPEDIFORMES & Podicipedidae (Bonaparte, 1831) & 2 & 0.0059 \\
\hline
\end{tabular}


Pizarro NEYRA, J. Predominancia ecológica y taxonómica de nombres de aves entre estudiantes universitarios peruanos. Ethnoscientia v. 6, n. 1, 2021. DOI: 10.22276/ethnoscientia.v6i1.353

\begin{tabular}{|c|c|c|c|c|}
\hline Chate (A) & PASSERIFORMES & $\begin{array}{l}\text { Leistes bellicosus (De Filippi, } \\
1847 \text { ) }\end{array}$ & 2 & 0.0059 \\
\hline Cormorán (A) & SULIFORMES & $\begin{array}{l}\text { Phalacrocorax brasilianus } \\
\text { (Gmelin, 1789); Phalacrocorax } \\
\text { bougainvilliii (Lesson, 1837); } \\
\text { Phalacrocorax gaimardi } \\
\text { (Lesson; Garnot, 1828) }\end{array}$ & 2 & 0.0059 \\
\hline Pinzon (A) & PASSERIFORMES & $\begin{array}{l}\text { Coryphospingus } \\
\text { (Muller, 1776) }\end{array}$ & 2 & 0.0059 \\
\hline Pato Pekin (M) & ANSERIFORMES & $\begin{array}{l}\text { Anas platyrrhynchus (Linnaeus, } \\
1758 \text { ) }\end{array}$ & 2 & 0.0059 \\
\hline Alondra (A) & PASSERIFORMES & Alaudidae (Vigors, 1825) & 2 & 0.0059 \\
\hline Tiptire (L) & STRIGIFORMES & $\begin{array}{l}\text { Athene cunicularia (Molina, } \\
\text { 1782) }\end{array}$ & 2 & 0.0059 \\
\hline Chuita (A) & SULIFORMES & $\begin{array}{l}\text { Phalacrocorax gaimardi } \\
\text { (Lesson \& Garnot, 1828) }\end{array}$ & 2 & 0.0059 \\
\hline $\begin{array}{l}\text { Loro cabeza azul } \\
\text { (M) }\end{array}$ & PSITTACIFORMES & $\begin{array}{l}\text { Pionus menstrus (Linnaeus, } \\
1766)\end{array}$ & 2 & 0.0059 \\
\hline Totorero (A) & PASSERIFORMES & $\begin{array}{l}\text { Phleocryptes melanops (Vieillot, } \\
\text { 1817) }\end{array}$ & 2 & 0.0059 \\
\hline Grato (A) & DESCONOCIDO & & 2 & 0.0059 \\
\hline Cotorra $(\mathrm{L})$ & PSITTACIFORMES & Psittacara wagleri (Gray, 1845) & 2 & 0.0059 \\
\hline Sinsonte (A) & PASSERIFORMES & $\begin{array}{l}\text { Mimus longicaudatus (Tschudi, } \\
\text { 1844) y otros Passeriformes }\end{array}$ & 2 & 0.0059 \\
\hline Mamani (A) & ACCIPITRIFORMES & $\begin{array}{l}\text { Gavilán, } \quad \text { Geranoaetus } \\
\text { melanoleucus (Vieillot, 1819) }\end{array}$ & 2 & 0.0059 \\
\hline Patosor $(\mathrm{A})$ & DESCONOCIDO & & 2 & 0.0059 \\
\hline Charizard (A) & PERSONAJE DE ANIME & & 2 & 0.0059 \\
\hline Tejedor $(\mathrm{A})$ & PASSERIFORMES & Ploceidae (Sundevall, 1836) & 2 & 0.0059 \\
\hline Agazapo (A) & DESCONOCIDO & & 2 & 0.0059 \\
\hline Calafate (A) & PASSERIFORMES & $\begin{array}{l}\text { Padda (Lonchura) oryzivora } \\
\text { (Linnaeus, 1758) }\end{array}$ & 2 & 0.0059 \\
\hline Agapacho (A) & DESCONOCIDO & & 2 & 0.0059 \\
\hline $\begin{array}{l}\text { Gallina de Guinea } \\
\text { (M) }\end{array}$ & GALLIFORMES & $\begin{array}{l}\text { Numida meleagris (Linnaeus. } \\
\text { 1758) }\end{array}$ & 2 & 0.0059 \\
\hline Emu (A) & RHEIFORMES & $\begin{array}{l}\text { Dromaeus novaehollandeiae } \\
\text { (Latham, 1790) }\end{array}$ & 2 & 0.0059 \\
\hline Ninfa (A) & DESCONOCIDO & & 2 & 0.0059 \\
\hline Alas azules $(\mathrm{M})$ & PSITTACIFORMES & $\begin{array}{ll}\text { Brotogeris } & \text { cyanoptera } \\
\text { (Salvadori, 1891) }\end{array}$ & 2 & 0.0059 \\
\hline Aguineo (A) & DESCONOCIDO & & 2 & 0.0059 \\
\hline Quilacoca (A) & DESCONOCIDO & & 2 & 0.0059 \\
\hline Charran $(\mathrm{A})$ & CHARADRIIFORMES & Sterna spp. (Linnaeus, 1758) & 2 & 0.0059 \\
\hline Chorlitejo (A) & CHARADRIIFORMES & $\begin{array}{l}\text { Charadrius spp. (Linnaeus, } \\
\text { 1758) }\end{array}$ & 2 & 0.0059 \\
\hline Batara (A) & PASSERIFORMES & $\begin{array}{l}\text { Thamnophilus bernardi (Lesson, } \\
\text { 1844) }\end{array}$ & 2 & 0.0059 \\
\hline Pancullo (A) & DESCONOCIDO & & 2 & 0.0059 \\
\hline Pihuicho (M) & PSITTACIFORMES & $\begin{array}{l}\text { Brotogeris versicolorus (Muller, } \\
1776 \text { ) }\end{array}$ & 2 & 0.0059 \\
\hline Diamante (A) & PSITTACIFORMES & Taeniopygia guttata (Vieillot, & 2 & 0.0059 \\
\hline
\end{tabular}


Pizarro NEYRA, J. Predominancia ecológica y taxonómica de nombres de aves entre estudiantes universitarios peruanos. Ethnoscientia v. 6, n. 1, 2021. DOI: 10.22276/ethnoscientia.v6i1.353

\begin{tabular}{|c|c|c|c|c|}
\hline & & 1817) & & \\
\hline Atrapamoscas (L) & PASSERIFORMES & $\begin{array}{ll}\text { "Flycatchers" } & \text { Tyrannidae } \\
\text { (Vigors, 1825) } & \\
\end{array}$ & 2 & 0.0059 \\
\hline Pato Jerga (A) & ANSERIFORMES & Anas georgica (Gmelin, 1789) & 2 & 0.0059 \\
\hline Raminegra (A) & DESCONOCIDO & & 2 & 0.0059 \\
\hline Paloma blanca (M) & COLUMBIFORMES & Columba livia (Gmelin, 1789) & 2 & 0.0059 \\
\hline Gallina china $(\mathrm{M})$ & GALLIFORMES & Gallus gallus (Linnaeus, 1758) & 2 & 0.0059 \\
\hline Agapornis (A) & PSITTACIFORMES & Agapornis spp. (Selby, 1836) & 2 & 0.0059 \\
\hline $\begin{array}{l}\text { Paloma Africana } \\
\text { (A) }\end{array}$ & COLUMBIFORMES & $\begin{array}{ll}\begin{array}{l}\text { Streptopelia } \\
\text { (Sundevall, 1857) }\end{array} & \text { roseogrisea } \\
\end{array}$ & 2 & 0.0059 \\
\hline Busardo (A) & ACCIPITRIFORMES & $\begin{array}{l}\text { Geranoaetus } \\
\text { (Vieillot, 1819) }\end{array}$ & 2 & 0.0059 \\
\hline Kakapo (A) & PSITTACIFORMES & Strigops habroptila (Gray, 1845) & 2 & 0.0059 \\
\hline Vencejo (L) & APODIFORMES & $\begin{array}{l}\text { Aeronautes andecolus } \\
\text { (d'Orbigny; Lafresnaye, } 1837 \text { ) }\end{array}$ & 2 & 0.0059 \\
\hline Chotacabras (L) & CAPRIMULGIFORMES & $\begin{array}{ll}\text { Chordeiles } & \text { acutipennis } \\
\text { (Hermann, 1783) }\end{array}$ & 2 & 0.0059 \\
\hline Frailecillo $(\mathrm{A})$ & CHARADRIIFORMES & $\begin{array}{l}\text { Fratercula arctica (Linnaeus, } \\
1758 \text { ) y otros Charadriiformes }\end{array}$ & 2 & 0.0059 \\
\hline Tangara (L) & PASSERIFORMES & Thraupidae (Cabanis, 1847) & 2 & 0.0059 \\
\hline Koel (A) & CUCULIFORMES & $\begin{array}{l}\text { Eudynamys orientalis (Linnaeus, } \\
1766 \text { ) }\end{array}$ & 2 & 0.0059 \\
\hline Soca (A) & GRUIFORMES & $\begin{array}{l}\text { Fulica ardesiaca (Tschudi, } \\
1843 \text { ) }\end{array}$ & 2 & 0.0059 \\
\hline Mosquetero (A) & PASSERIFORMES & Phylloscopus spp. & 2 & 0.0059 \\
\hline Turtupilin (L) & PASSERIFORMES & $\begin{array}{l}\text { Pyrocephalus rubinus (Boddaert, } \\
\text { 1783) }\end{array}$ & 2 & 0.0059 \\
\hline Canastero (A) & PASSERIFORMES & $\begin{array}{l}\text { Asthenes } \\
\text { (Reichenbach, 1853) y otros } \\
\text { Furnariidae }\end{array}$ & 2 & 0.0059 \\
\hline Comesebo (L) & PASSERIFORMES & $\begin{array}{l}\text { Conirostrum } r \text { cinereum } \\
\text { (Lafresnaye; D'Orbigny, 1838); } \\
\text { Troglodytes aedon (Vieillot, } \\
1809 \text { ) }\end{array}$ & 2 & 0.0059 \\
\hline Arrendajo (A) & PASSERIFORMES & $\begin{array}{l}\text { Garrulus glandarius (Linnaeus, } \\
1758 \text { ) }\end{array}$ & 2 & 0.0059 \\
\hline Pecho rojo (A) & PASSERIFORMES & $\begin{array}{l}\text { Leistes bellicosus (De Filippi, } \\
1847 \text { ) }\end{array}$ & 2 & 0.0059 \\
\hline Pisaka (A) & TINAMIFORMES & $\begin{array}{l}\text { Nothoprocta ornata (Gray, } \\
\text { 1867) }\end{array}$ & 2 & 0.0059 \\
\hline $\operatorname{Huacacho}(\mathrm{A})$ & PELECANIFORMES & Pelecanus thagus (Molina, 1782) & 2 & 0.0059 \\
\hline Pardela (A) & PROCELLARIFORMES & $\begin{array}{l}\text { Ardenna grisea (Gmelin, 1789) y } \\
\text { otros Procellariformes }\end{array}$ & 2 & 0.0059 \\
\hline Huntir (A) & DESCONOCIDO & & 2 & 0.0059 \\
\hline Gallareta (A) & GRUIFORMES & $\begin{array}{l}\text { Gallinula galeata (Lichtenstein, } \\
\text { 1818); Fulica ardesiaca } \\
\text { (Tschudi, 1843) }\end{array}$ & 2 & 0.0059 \\
\hline Gaviota gris $(\mathrm{A})$ & CHARADRIIFORMES & Larus modestus (Tschudi, 1843) & 2 & 0.0059 \\
\hline Gaviota roja $(\mathrm{A})$ & CHARADRIIFORMES & No identificado & 2 & 0.0059 \\
\hline J'uku (L) & STRIGIFORMES & Bubo virginianus (Gmelin, 1788) & 2 & 0.0059 \\
\hline
\end{tabular}


Pizarro NEYRA, J. Predominancia ecológica y taxonómica de nombres de aves entre estudiantes universitarios peruanos. Ethnoscientia v. 6, n. 1, 2021. DOI: 10.22276/ethnoscientia.v6i1.353

\begin{tabular}{|c|c|c|c|c|}
\hline Puku-puku (A) & CHARADRIIFORMES & $\begin{array}{l}\text { Thinocorus orbignyianus } \\
\text { (Geoffroy Saint-Hilaire; Lesson, } \\
\text { 1831) }\end{array}$ & 2 & 0.0059 \\
\hline Putilla (L) & PASSERIFORMES & $\begin{array}{l}\text { Pyrocephalus rubinus (Boddaert, } \\
1783 \text { ) }\end{array}$ & 2 & 0.0059 \\
\hline Chorlo (A) & CHARADRIIFORMES & Calidris spp. (Merrem, 1804) & 2 & 0.0059 \\
\hline Zarapito (A) & CHARADRIIFORMES & $\begin{array}{l}\text { Numenius phaeopus (Linnaeus, } \\
1758 \text { ) }\end{array}$ & 2 & 0.0059 \\
\hline Kili-kili (L) & FALCONIFORMES & $\begin{array}{lll}\text { Falco } & \text { sparverius } & \text { (Linnaeus, } \\
1758)\end{array}$ & 2 & 0.0059 \\
\hline Trogon (A) & TROGONIFORMES & Trogonidae (Lesson, 1828) & 1 & 0.0029 \\
\hline Paca paca $(\mathrm{L})$ & STRIGIFORMES & $\begin{array}{l}\text { Glaucidium peruanum (König, } \\
\text { 1991) }\end{array}$ & 1 & 0.0029 \\
\hline Avutarda (A) & OTIDIDAE & Otididae (Rafinesque, 1815) & 1 & 0.0029 \\
\hline Gallina ciega $(\mathrm{L})$ & CAPRIMULGIFORMES & $\begin{array}{ll}\begin{array}{l}\text { Systellura } \\
\text { (Bonaparte, 1825) }\end{array} & \text { longirostris } \\
\end{array}$ & 1 & 0.0029 \\
\hline $\begin{array}{l}\text { Aguila pescadora } \\
\text { (A) }\end{array}$ & ACCIPITRIFORMES & $\begin{array}{l}\text { Pandion haliaetus (Linnaeus, } \\
\text { 1758). }\end{array}$ & 1 & 0.0029 \\
\hline Pajaro niño (A) & SPHENISCIFORMES & $\begin{array}{l}\text { Spheniscus humboldti (Meyen, } \\
\text { 1834) }\end{array}$ & 1 & 0.0029 \\
\hline Chorlito gris (A) & CHARADRIIFORMES & Calidris spp. (Merrem, 1804) & 1 & 0.0029 \\
\hline Aguila blanca (L) & ACCIPITRIFORMES & $\begin{array}{l}\text { Geranoaetus polyosoma (Quoy; } \\
\text { Gaimard, 1824) }\end{array}$ & 1 & 0.0029 \\
\hline Pajaro negro (L) & PASSERIFORMES & $\begin{array}{l}\text { Molothrus bonariensis (Gmelin, } \\
1789 \text { ) }\end{array}$ & 1 & 0.0029 \\
\hline Cinclodes (A) & PASSERIFORMES & Cinclodes spp. (Gray, 1840) & 1 & 0.0029 \\
\hline Polla de agua (A) & GRUIFORMES & $\begin{array}{l}\text { Gallinula galeata (Lichtenstein, } \\
\text { 1818). }\end{array}$ & 1 & 0.0029 \\
\hline Avefría (A) & CHARADRIIFORMES & $\begin{array}{l}\text { Vanellus respledens (Tschudi, } \\
\text { 1843). }\end{array}$ & 1 & 0.0029 \\
\hline $\begin{array}{l}\text { Pato } \text { Merganetta } \\
\text { (A) }\end{array}$ & ANSERIFORMES & $\begin{array}{l}\text { Merganetta armata (Gould, } \\
\text { 1842). }\end{array}$ & 1 & 0.0029 \\
\hline $\begin{array}{l}\text { Lechucita bigotona } \\
\text { (A) }\end{array}$ & STRIGIFORMES & $\begin{array}{l}\text { Xenoglaux loweryi } \quad \text { (O’Neil; } \\
\text { Graves, 1977) }\end{array}$ & 1 & 0.0029 \\
\hline
\end{tabular}

*(A) = ausente; $(\mathrm{M})$ = especie manejada o asilvestrada; (L) = especie de distribución local. Fuente: el autor (2020). 